\title{
COMPATIBILITY ISSUES IN VLBI
}

\author{
R. D. WIETFELDT
}

Space Geodynamics Laboratory, Institute of Space and Terrestrial Science, 2700 Steeles Ave. West, Concord, Ontario, Canada L4K 3C8

L. R. D'ADDARIO

National Radio Astronomy Observatory, 2015 Ivy Road, Charlottesville, Virginia 22903, USA

\begin{abstract}
New VLBI systems are under development in many countries in preparation for large dedicated arrays, including orbiting radio telescopes to be launched in the 1990s. In particular, several new recording systems and correlators are being constructed: the VLBA in the U.S., the S2 in Canada, and the K4/VSOP in Japan. This paper reviews the issues of technical compatibility among these systems in an effort to facilitate global VLBI experiments.
\end{abstract}

\section{INTRODUCTION}

VLBI experiments have been conducted worldwide with more than 30 radio telescopes participating. The success of such experiments has depended on the existence of sufficiently large correlators to process the data and on the ability of the various telescopes to record data in a form that can be reproduced and crosscorrelated on those machines. This has required widespread standardization of all the signal processing, including choice of frequency, polarization, bandwidth, digitization, and calibration methods, as well as recording methods.

Sensitivity in continuum measurements has been limited by the bandwidth that can be recorded and processed, so over the years there has been an evolution toward larger bandwidth. We are now on the threshold of the next generation of recording systems, in which the maximum bandwidth and the recording density have been significantly increased. Unlike the previous situation in which only one design existed at a given performance level, there are now several different systems with similar performance. These include the VLBA, which uses multi-track longitudinal recording (achieving $256 \mathrm{Mb} / \mathrm{s}$ for $6 \mathrm{~h}$ per tape); the S2 (Canadian) system, which uses an array of 8 video cassette recorders $(128 \mathrm{Mb} / \mathrm{s}$, $6 \mathrm{~h}$ ); and the K4 (Japanese) system, which uses a large-format helical scan cassette under an ANSI standard called ID-1 $(256 \mathrm{Mb} / \mathrm{s}, 1 \mathrm{~h})$. The latter two are being developed partly in support of the VLBI spacecraft known as Radioastron (USSR) and VSOP (Japan). It is therefore timely to ask: Can the various systems be made to operate together in some sense? Is there some further effort toward standardization that might be undertaken for future systems? 


\section{GENERAL REQUIREMENTS}

A block diagram of a generic VLBI signal processing system is shown in Fig. 1 . The boundaries of the blocks have been defined with some care, so that various technologies and existing designs can be described in terms of similar interface signals. In all existing VLBI systems, the "transmission medium" is a magnetic tape; but the generic system allows it to be a waveguide, an optical fiber, a radio link, or some other technology.

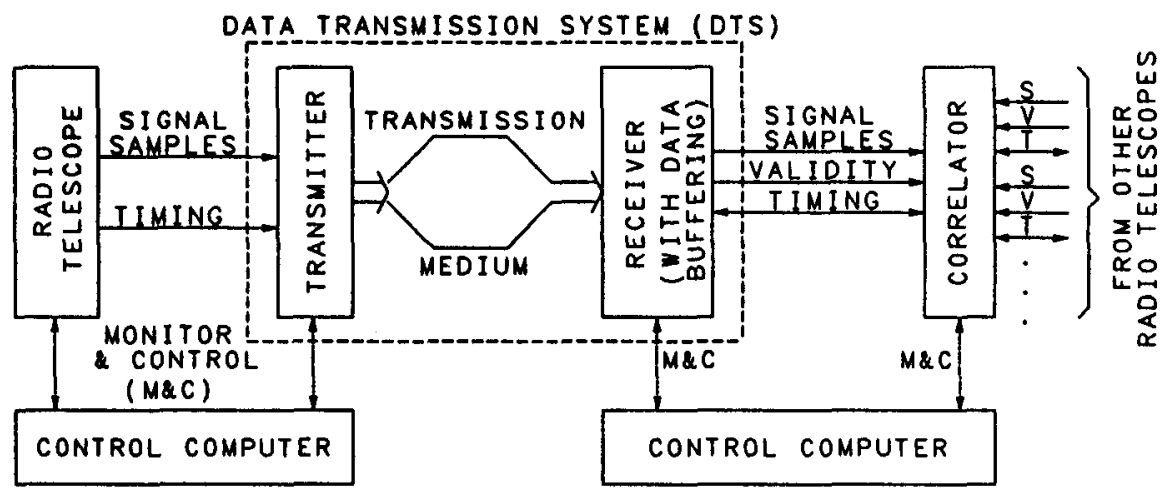

EIGURE 1. GENERIC VLBI DAIA HANDLING

The radio telescope is assumed to produce astronomical signals in digitized form. The telescope must also provide timing information with sufficient resolution and range that the time of day (say, Universal Time) of each signal sample is available to the "data transmission system."

The data transmission system consists of a "transmitter" (e.g., a recorder), a "medium" (e.g., tape), and a "receiver" (e.g., a playback machine). The system must reproduce the signal samples at its output and must also recover the time at which each sample was taken. The receiver will usually contain some temporal buffering so as to allow the data times to be synchronized, at least roughly, among the telescopes. In general, the data will not be reproduced perfectly, and the receiver may be able to determine that certain blocks of data contain an excessive number of errors; this information should be included in the output in the form of validity signals.

The "correlator" in Fig. 1 includes only those functions which are independent of the transmission system technology, and hence might be called the "correlator proper." In some implementations, functions of the transmission system (such as decoding or error detection) might be integrated with the correlator so that there is no physical interface of the form depicted, but this is not the case for recent designs. The correlator must provide timing information to all data receivers. The details of this time synchronization are the main differences among correlator designs with respect to their input interfaces.

At each telescope and at each data receiver a low-speed link to a computer is assumed to be available for passing control and monitor information whose timing is much less critical than one sample time. The slower part of the sample time information might also be passed across these links. 


\section{CHANNELIZATION AND DIGITIZATION}

For many experiments, the telescope signals are naturally separated into several channels. For example, it is often desired to observe both orthogonal polarizations, sometimes with receivers covering two separate bands. For continuum observations, sensitivity is dependent on the total bandwidth processed, and a given bandwidth can be achieved in many narrow channels or a few wide channels; modern designs favor the latter because it minimizes the cost, mass, and power consumption of the pre-digitization electronics.

The selection of center frequency, bandwidth, polarization, and sideband (upper or lower) for each channel will be called the "channelization" of the telescope. The processing of an experiment is simplified if the channelizations at all telescopes are identical, and this has been a firm requirement for all experiments to date. However, the development of "FX" correlators, where each channel's signal is first converted to the frequency domain by an FFT before being cross-correlated with the signals from other telescopes, makes it possible to accommodate slightly different channelizations. A single wide bandwidth channel from one telescope can be correlated against several narrower bandwidth channels from another if the latter are contiguous and correspond to the same signal frequencies, polarization, and sideband as the former. This is accomplished by selecting the FFT lengths so that their outputs correspond to the same observing frequencies for each telescope.

Channelizations planned for the VLBA, VSOP, and Radioastron are not identical. VSOP's channel bandwidths are 64,32 or $16 \mathrm{MHz}$; VLBA's are 16 $\mathrm{MHz}$ to $.062 \mathrm{MHz}$ by factors of 2; and Radioastron's are 8,4 , and $2 \mathrm{MHz}$. The maximum numbers of channels are 2, 8 (expandable), and 8 , respectively. The VSOP and VLBA correlators will accommodate some non-native bandwidths by the method described above, easing the compatibility problem somewhat.

Digitization involves quantization and sampling. All VLBI experiments to date have involved the simplest possible quantization, namely 2-level.Finer quantization improves sensitivity per unit observing time, so new designs (VLBA, S2, K4) are supporting 4-level quantization. A 4-level correlator supports 2-level as a special case, and a 2-level correlator can be made to process 4-level data in four passes; furthermore, cross correlation between 2-level and 4-level signals is straightforward. Thus, these two quantizations can be considered compatible. Nominal sampling rates in VLBI have long been standardized at $2^{K} \mathrm{MHz}$ for integer $K$, and there seems to be no reason to depart from this.

\section{RECORDING AND REPRODUCING}

In view of the various recording and reproducing systems now existing or being developed, we inquire whether recordings made at a telescope using one system can be processed at a correlator designed for another.

To achieve direct transfer of the recorded medium from one system to the other, we must have (a) compatible media (dimensions and magnetic characteristics), (b) the same physical arrangement of data on the medium, and (c) a reproducing system which can accept the encoding used by the recording system (including the embedded timing information). These are severe restrictions that are unlikely to be met except by systems using nearly the same technology. The 
VLBA, S2, and K4 systems have mutually incompatible media. Future systems are likely to be incompatible at this level with present and earlier ones.

Another level of compatibility can be achieved if recorders or reproducers of different types can be readily substituted for each other at any telescope or correlator, respectively. This would be greatly facilitated if the telescopeto-transmitter interface and the receiver-to-correlator interface (see Fig. 1) were standardized. Such standardizations should be based on the idea that the signals passing across each interface be independent of the transmission technology.

\section{THE CORRELATOR INTERFACE}

We consider here several options for connecting a "foreign" receiver to a particular correlator, in substitution for its "native" receiver.

Direct. The connection is simplest if it can be made at the input of the correlator proper, where the data is least dependent on the transmission technology. If the foreign and native receivers are standardized at this interface, then direct connection is possible; if they are similar, then connection through an adapter may be possible.

Reformatting. Some correlators have been designed so that the input to the correlator proper is not accessible. In that case, the foreign receiver's output might be decoded and then re-encoded to resemble that seen at an intermediate interface of the native receiver.

Copying. Regardless of the interface designs, it is generally possible to build an off-line machine to copy a foreign recording onto another medium in native form. This requires one foreign receiver and one native transmitter in addition to the native receivers needed by the correlator. It also requires converting the foreign output interface to conform to the native input interface, but this is relatively easy if it is done with decoded ("unformatted") data. There is no need for synchronization of the foreign receiver with native receivers or with the correlator, as there is for the other options. Most importantly, a single copying machine allows the correlator to accept data from any number of foreign telescopes, provided that the total volume of foreign data is sufficiently small. In that circumstance this option is strongly favored, even if the other options are feasible.

It is worthwhile to look at the correlator input interfaces of the three systems on which we are concentrating: VLBA, S2, and VSOP/K4. One of the main difficulties is the timing interface, because the time synchronization task has been divided between the two sides of this interface in different ways. In all three systems, the tape speed is adjusted so that data corresponding to a desired time will be presented to the correlator. In the VLBA, the desired time is computed precisely by the correlator (in a "delay model generator") and sent frequently to the receiver, which must supply data to the nearest sample; it does this by internally adjusting the tape speed and maintaining a FIFO buffer sufficient to absorb mechanical errors. The S2 is similar, except that timing commands are sent less frequently ( 1 per second); the actual time of the data is available to the correlator, but it must include buffering for fine adjustment of the time. In $\mathrm{K} 4$ (Type 0), the receivers are able to synchronize to each other and deliver data at a common time; the correlator must then provide all timing offsets. 